\title{
ARTICLE
}

Epidemiology and Population Health

\section{Obesity is a potential risk factor contributing to clinical manifestations of COVID-19}

\author{
Zhenyu Kang ${ }^{1} \cdot$ Shanshan Luo $^{2} \cdot$ Yang Gui ${ }^{1} \cdot$ Haifeng Zhou ${ }^{1} \cdot$ Zili Zhang $^{1} \cdot$ Chunxia Tian $^{1} \cdot$ Qiaoli Zhou ${ }^{1}$. \\ Quansheng Wang ${ }^{1} \cdot \mathrm{Yu} \mathrm{Hu}^{2} \cdot$ Heng Fan ${ }^{1} \cdot$ Desheng $\mathrm{Hu} \mathbb{D}^{1,2}$
}

Received: 7 April 2020 / Revised: 2 August 2020 / Accepted: 3 September 2020 / Published online: 13 September 2020

(c) The Author(s), under exclusive licence to Springer Nature Limited 2020

\begin{abstract}
Background Since December 2019, novel coronavirus (SARS-CoV-2)-induced pneumonia (COVID-19) occurred in Wuhan, and rapidly spread throughout China. COVID-19 patients demonstrated significantly different outcomes in clinic. We aimed to figure out whether obesity is a risk factor influencing the progression and prognosis of COVID-19.

Methods 95 patients with COVID-19 were divided into obesity group and non-obesity group according to their body mass index (BMI). The demographic data, clinical characteristics, laboratory examination, and chest computed tomography (CT) were collected, analyzed and compared between two groups.

Results Our data showed that COVID-19 patients with obesity had more underlying diseases and higher mortality rate compared to those without obesity. Furthermore, patients with obesity also demonstrated more severe pathological change in lung and higher blood lymphocytes, triglycerides, IL-6, CRP, cystatin C, alanine aminotransferase (ALT), erythrocyte sedimentation rate (ESR), which may greatly influence disease progression and poor prognosis of COVID-19.

Conclusions It suggest that obesity contributes to clinical manifestations and may influence the progression and prognosis of COVID-19 and it is considered as a potential risk factor of the prognosis of COVID-19. Special medical care and appropriate intervention should be performed in obesity patients with COVID-19 during hospitalization and later clinical follow-up, especially for those with additional other comorbidities.
\end{abstract}

\section{Introduction}

In December 2019, a novel coronavirus (SARS-CoV-2) induced pneumonia, named COVID-19 by World Health

These authors contributed equally: Zhenyu Kang, Shanshan Luo, Yang Gui

Supplementary information The online version of this article (https:// doi.org/10.1038/s41366-020-00677-2) contains supplementary material, which is available to authorized users.

Desheng $\mathrm{Hu}$

desheng.hu@ @ust.edu.cn

1 Department of Integrated Traditional Chinese and Western Medicine, Union Hospital, Tongji Medical College, Huazhong University of Science and Technology, Wuhan 430022, China

2 Institute of Hematology, Union Hospital, Tongji Medical College, Huazhong University of Science and Technology, Wuhan 430022, China
Organization (WHO), outbroken in Wuhan, China. SARSCoV-2 can transmit from person to person through contact or respiratory aerosols. Patients with COVID-19 showed acute respiratory symptoms similar to the symptoms from the patients with SARS-CoV infection, and had an incubation period of about 3-14 days [1-3]. The evolution analysis showed that SARS-CoV-2 belongs to the $\beta$ genus of coronavirus and has $96 \%$ homology with a bat-derived SARS-like virus $[2,4]$. In vitro study confirmed that SARS$\mathrm{CoV}-2$ enter the host via its $\mathrm{S}$ protein binding to cell surface receptor ACE2 [4-6].

Most patients with COVID-19 are mildly ill and have a flu-like upper respiratory symptoms, such as fever, dry cough, fatigue, and muscle soreness and so on. However, some patients may progress to acute respiratory distress syndrome, metabolic acidosis, or coagulation dysfunction, even leading to death $[1,2,7,8]$. Thus, the duration of hospitalization of COVID-19 patient varies from person to person. Knowing the factors causing such clinical 
difference can guide people to take right cares to protect infection and guide clinic treatment.

It was estimated by WHO that over $50 \%$ of the current global population is overweight or obese, which is often accompanied with more underlying diseases [9]. Increasing evidences suggested that obesity may increase the risk of infection and affect the courses of disease [10-13]. Studies demonstrated that obesity can increase the ability of influenza virus replication and variation, also promote the emergence of potential mutant strains emergence, and it greatly influenced the mortality rate, viral titers in lung and worsened lung pathology [9, 14]. Moreover, recent publication indicated that the obesity patients with COVID-19 demonstrated a high frequency of intensive care and greater disease severity [15]. The presence of obesity in patients with metabolic associated fatty liver disease was associated with increased risk of severe COVID-19 illness [16]. Type 2 diabetes mellitus, hypertension, and cardiovascular diseases were frequently detected in subjects who died from COVID -19 in Italy [17, 18]. It can be inferred that obesity-related comorbidities could represent an unfavorable factors for complications of COVID-19 in obesity, which might greatly influenced the proliferation of viruses, tissue damage, and further affected mortality rate.

In order to figure out whether obesity is a risk factor for the prognosis of COVID-19, we studied 95 admitted COVID-19 cases in Wuhan Union Hospital and divided them into obesity and non-obesity groups based on body mass index (BMI). Basic information, chest CT scans and laboratory examination were collected and analyzed. Our data showed that obesity is a risk factor and associated with poor prognosis of COVID-19.

\section{Methods}

\section{Study design and participants}

95 admitted patients with COVID-19 in Wuhan Union Hospital from February 15 to March 02, 2020 were enrolled in this retrospective single-center study. Patients were divided into two groups, patients with BMI scores greater than or equal to 25 were classified as obesity patients according to the International Association for the Study of Obesity, the International Obesity Task Force, and the WHO for the recommended cut off points for Asian populations, and the rest were non-obesity patients [19-21]. This case series was approved by the Ethics Committee of Wuhan Union Hospital, Tongji Medical College, Huazhong University of Science and Technology (No. [2020]93). Written informed consent was waived by the Ethics Commission of the designated hospital for the emerging infectious diseases.

\section{Procedures}

We collected 95 patients and analyzed their demographic data, medical history, contact history, signs and symptoms, laboratory test results, and chest CT scans from the electronic medical record. The date of the onset of the disease is defined as the date of the first symptom. The clinical results were followed up to March 15, 2020. Laboratory verification of SARS-CoV-2 was performed at Wuhan Union Hospital. Throat-swab specimens obtained from the patient's upper respiratory tract were kept in viral-transport medium. Total RNA was extracted in $2 \mathrm{~h}$ using a respiratory sample RNA isolation kit (Zhongzhi, Wuhan, China). SARS-CoV-2 nucleic acid was examined by RT-PCR as previously described [22]. In addition, CT imaging scores were used to quantify the pathological changes of the lung from patients with COVID-19. These values were obtained by two physicians with an introduced scoring system as described in Table 1 by randomized double blind.

\section{Statistical analysis}

Categorical variables are expressed as frequency and percentage (\%), continuous variables were expressed as mean (SD) if they are normally distributed or median (IQR) if not. Categorical variables were compared between the two groups using the $\chi^{2}$ test or Fisher's exact test, and continuous variables were analyzed using the two-sided Student's $t$ test or the Mann-Whitney $U$ test as appropriate. A $P$ value of $<0.05$ was considered statistically significant. Estimate of variation within each group was analyzed and groups showed similar variance were compared. The power calculations were performed in the same way as in the original study [23]. Power $>0.75$ represent the $P>0.05$ group is indeed not statistically significants, vice versa. All statistical analyses were performed using SPSS 20.0 software.

Table 1 The scoring system of CT imaging in patients with COVID-19.

\begin{tabular}{lll}
\hline No. & Performance & Score \\
\hline (1) & Unbilateral patchy shadows or ground glass opacity & 5 \\
(2) & Bilateral patchy shadows or ground glass opacity & 7 \\
(3) & Diffuse changes for (1) or (2) & 2 \\
(4) & Unbilateral solid shadow, strip shadow & 2 \\
(5) & Bilateral solid shadow, strip shadow & 4 \\
(6) & Unbilateral Pleural effusion & 2 \\
(7) & Bilateral pleural effusion & 4 \\
(8) & Increased or enlarge mediastinal lymph nodes & 1 \\
\hline
\end{tabular}




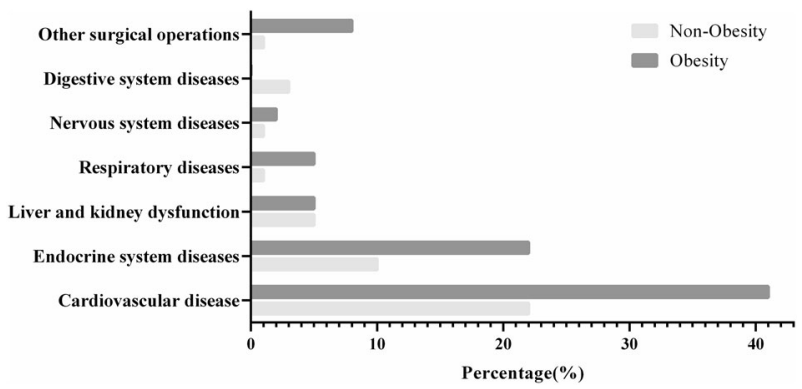

Fig. 1 Comparison of basic diseases between obesity and nonobesity patients. The percentage of basic diseases distribution in obesity and non-obesity patients was statistically analyzed.

\section{Results}

Demographics data showed obesity patients have more underlying diseases such as cardiovascular diseases $(41.7 \%$ vs. $22.0 \%)$, endocrine system abnormalities $(22.2 \%$ vs. $10.2 \%)$, respiratory diseases $(5.6 \%$ vs. $1.7 \%)$, and neurological diseases (2.8\% vs. $1.7 \%$ ) compared to non-obesity group (Fig. 1, Supplementary Table S1). There was no significant difference in age between obesity patients and non-obesity patients (52 [IQR, 21-81] vs. 53 [IQR, 24-84]) (Supplementary Table S1). Gender and other baseline symptoms between the two groups and the time from symptom onset to hospitalization were analyzed (Supplementary Table S1). For all patients, the most common symptoms are fever (46.3\%), cough (21.1\%), fatigue $(15.8 \%)$, and diarrhea $(13.7 \%)$, whereas chest tightness $(9.5 \%)$, myalgia $(8.4 \%)$, sputum production $(5.3 \%)$, headache $(5.3 \%)$, chest pain (4.2\%), nausea and vomiting $(4.2 \%)$, chill (3.2\%), shortness of breath $(2.1 \%)$, and izziness $(1.1 \%)$ were relatively rare. Frequency of cough $(36.1 \%$ vs. $11.9 \%)$ and fatigue $(33.3 \%$ vs. $5.1 \%)$ in obesity patients was much higher compare to non-obesity patients. Mortality rates of the patients was relatively higher in obesity ones (5.5\% vs. 0\%) suggesting a worse outcome of SARS-CoV-2 infection (Supplementary Table S1).

At admission, abnormal chest CT images were detected in all patients. We compared the representative chest CT imaging of obesity and non-obesity patients and found that the distribution and frequency of lung lesions were different (Fig. 2a, Supplementary Table S2). All patients with COVID-19 displayed varying degrees of typical groundglass opacities in lung (Fig. 2b). The lung lesions in obesity patients were more widely distributed as manifested random $(83.3 \%$ vs. $61.0 \%)$, while the non-obesity patients showed peripheral infection of lung more frequency $(33.9 \%$ vs. $11.1 \%$ ). $91.7 \%$ obesity patients performed bilateral multilobe lesions while non-obesity patients showed $84.7 \%$. The obesity ones also manifested higher proportion of specific ground-glass opacity (100.0\% vs. $94.9 \%)$, crazy-paving pattern $(30.6 \%$ vs. $23.7 \%)$, enlarged or increased number of pulmonary mediastinal lymph nodes (33.3\% vs. $10.2 \%)$, and pleural effusion (11.1\% vs. $10.2 \%)$, which represent more severe lung lesions (Fig. 2a, Supplementary Table S2). In addition, the severity of lung pathological changes was scored with reference to the criteria (Table 1). It shows that the score of CT imaging for patients with obesity was higher, in comparison to the one for non-obesity patients (Fig. 2c). It suggest that lung injury was more severe in obesity patients, which represents worse disease progression.

According to statistical results, the values of some laboratory tests for all patients are abnormal (Supplementary Table S3). For both groups, inflammation-related markers such as IL-6 (5.84 [IQR, 2.95-108.89]), IL-4 (3.62 [IQR, 1.37-6.88]), hypersensitive C-reactive protein (hsCRP) (7.97 [IQR, 1.67-91.4]), and erythrocyte sedimentation rate (ESR) (28.5 [IQR, 2-112]), as well as enzyme alanine aminotransferase (ALT) (39 [IQR, 8-176]) and cystatin C (1.03 [IQR, 0.56-5.16]), were all significantly elevated. Monocyte count $\left(0.60 \times 10^{9} \quad[\mathrm{IQR}\right.$, $0.2-1.23]$ ) in two groups were slightly increased. IL-10 (5.13 [IQR, 2.19-10.14]) also showed a certain increase in all patients. However, the albumin/globulin (ALB/GLB) (1.4 [IQR, 0.8-2.3]) was significantly decreased in two groups. Low-density lipoprotein (LDL) (2.65 [IQR, 2.04-4.42]) also showed irregular. The lymphocytes $\left(1.15 \times 10^{9}\right.$ [IQR, 0.5-2.81]) in all patients showed abnormal changes. Triglycerides (1.5 [IQR, 0.3-7.59]) also manifested diversity. Creatine kinase (52 [IQR, 26-268]), fibrinogen (FIB) (3.78 [IQR, 2.05-8.69]), and D-dimer (0.26 [IQR, 0.2-2.11]) displayed no obvious abnormalities in two groups (Figs. 3, 4).

Further we compared the differences in laboratory tests between the obesity patients and non-obesity patients (Figs. 3, 4, Supplementary Table S3). It shows that for the obesity patients, triglycerides (1.81 [IQR, 0.63-6.43] vs. 1.34 [IQR, 0.3-7.59]), LDL (3.14 [IQR, 2.0-4.42] vs. 2.56 [IQR,2.04-4.27]), ALT (44 [IQR, 13-176] vs. 39 [IQR, 8-122]), cystatin C (1.21 [IQR, 0.56-5.16] vs. 0.95 [IQR, 0.57-1.89]), and inflammation-related markers such as hsCRP (12.8 [IQR, 4.17-91.4] vs. 6.61 [IQR, 1.67-40.12]), $\operatorname{ESR}(49$ [IQR, 2-112] vs. 25 [IQR, 2-68]), IL-6 (10.81 [IQR, 3.01-108.89] vs. 5.56 [IQR, 2.95-39.88]), and IL-4 (4.2 [IQR, 1.58-6.88] vs. 3.45 [IQR, 1.37-5.99]) were elevated to a much higher extent, compared to non-obesity patients (Fig. 3b, Fig. 4a). The count of monocyte $(0.64 \times$ $10^{9}$ [IQR, $0.2-1.23$ ] vs. $0.56 \times 10^{9}$ [IQR, 0.24-0.85]) was also superior in obesity group (Fig. 3a). IL-10 (5.21 [IQR, 2.23-9.02] vs. 5.13 [IQR, 2.19-10.14]) manifested higher level in obesity patients (Fig. 4a). Whereas, the ALB/GLB (1.2 [IQR, 0.8-2.0] vs. 1.06 [IQR, $1.4(1.0-2.3)]$ ]) was significantly lower in obesity patients (Fig. 3b). Abnormal changes were also found in absolute count of lymphocytes 
Fig. 2 CT result in the obesity patients and non-obesity patients. a The frequency of lung injury distribution of the obesity and non-obesity patients. b The representative CT images of the obesity and non-obesity patients patients. c The CT score of the obesity and non-obesity patients. $P<0.05$ was considered statistically significant.

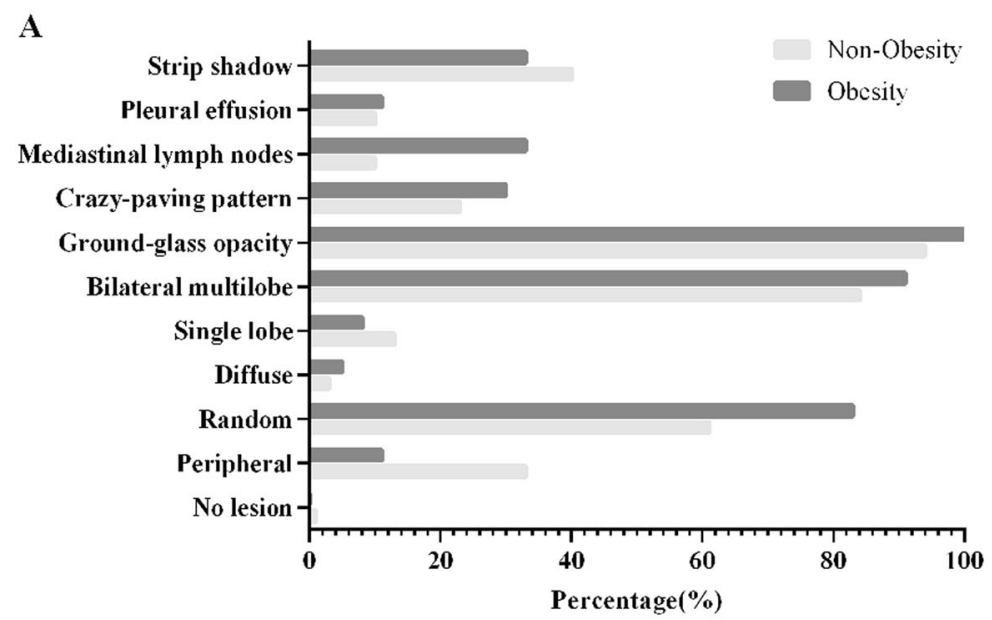

B
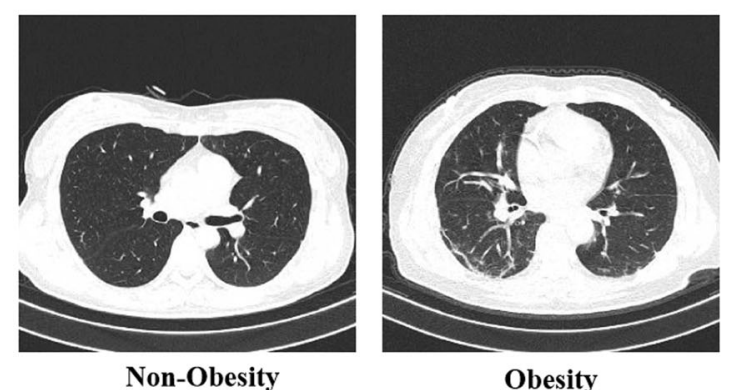

Obesity
C

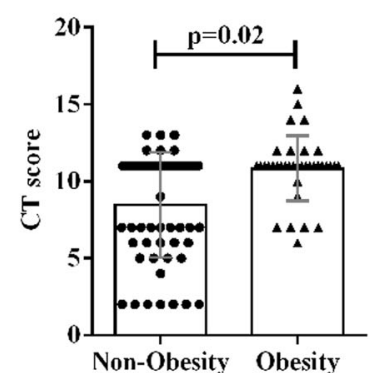

$\left(1.63 \times 10^{9} \quad[\mathrm{IQR}, \quad 0.5-1.57] \quad\right.$ vs. $1.06 \times 10^{9} \quad$ [IQR, $0.66-2.81]$ ) between two groups (Fig. 3a). In obesity group, creatine kinase (66.5 [IQR, 29-268] vs. 47 [IQR, 26-222]), uric acid (328.1 [IQR, 177.7-532] vs. 270.75 [IQR, 194-493]), and blood glucose (5.61 [IQR, 4.2-14.51] vs. 4.86 [IQR, 3.95-13.49]) performed higher trend in the normal range (Fig. 3b). The coagulation related factors FIB (3.87 [IQR, 2.58-8.69] vs. 3.60 [IQR, 2.05-6.83]) and Ddimer (0.36 [IQR, 0.2-2.11] vs. 0.22 [IQR, 0.2-2.11]) results were also a little higher in obesity patients than in non-obesity patients in the normal range (Fig. 4b). These data indicate that the abnormally elevated blood lipids, higher tissue injury-related enzyme release, excessive uncontrolled inflammatory response may greatly influence disease progression and lead to a poorer prognosis for COVID-19.

\section{Discussion}

In this study, we collected data from 95 cases with COVID19. Data showed that there was no significantly difference in age and gender between obesity and non-obesity patients. However, obesity patients have more underlying diseases such as cardiovascular disease and abnormal endocrine metabolism including diabetes and thyroncus as previous studies. These patients manifested more severe clinical symptoms, lung injury, elevated tissue damage and inflammation related parameters, and higher mortality rate compared to non-obesity ones.

As it reported, obesity is the result of abnormal energy metabolism, leading to weight gain and metabolic disorders, which in turn cause tissue stress and dysfunction [24, 25]. In our study, obesity patients showed higher levels of triglycerides, LDL and blood glucose than non-obesity ones, this metabolic abnormality may greatly influence disease progression and poor prognosis of COVID-19. Obesity provide a unique microenvironment for the pathogenesis of the disease, and is characterized by a chronic, lowinflammatory state, which may lead to the production of exhausted immune cells, and the organism becomes more vulnerable to infections and less responsive to vaccinations, antivirals, and antimicrobial drugs [15, 26]. Chronic inflammation in obesity is characterized by a condition of adipocyte hypoxia and dysfunction, that results in an exuberant secretion of pro-inflammatory cytokines and the recruitment of immune cells including macrophages, T-cells and B-cells, and ultimately an auto-regenerating inflammation loop will be formed [26, 27].

An excessive immune response to SARS-CoV-2 is thought to be the major reason of disease severity and death for patients with COVID-19 [28]. The circulating proinflammatory stimuli, for example viral pathogen-associated molecular patterns trigger activation of blood monocytes, 
Fig. 3 Blood test and biochemical examination results of the obesity patients and non-obesity patients. a Blood test results of the obesity and non-obesity patients. b Biochemical examination results of the obesity and nonobesity patients. $P<0.05$ was considered statistically significant.
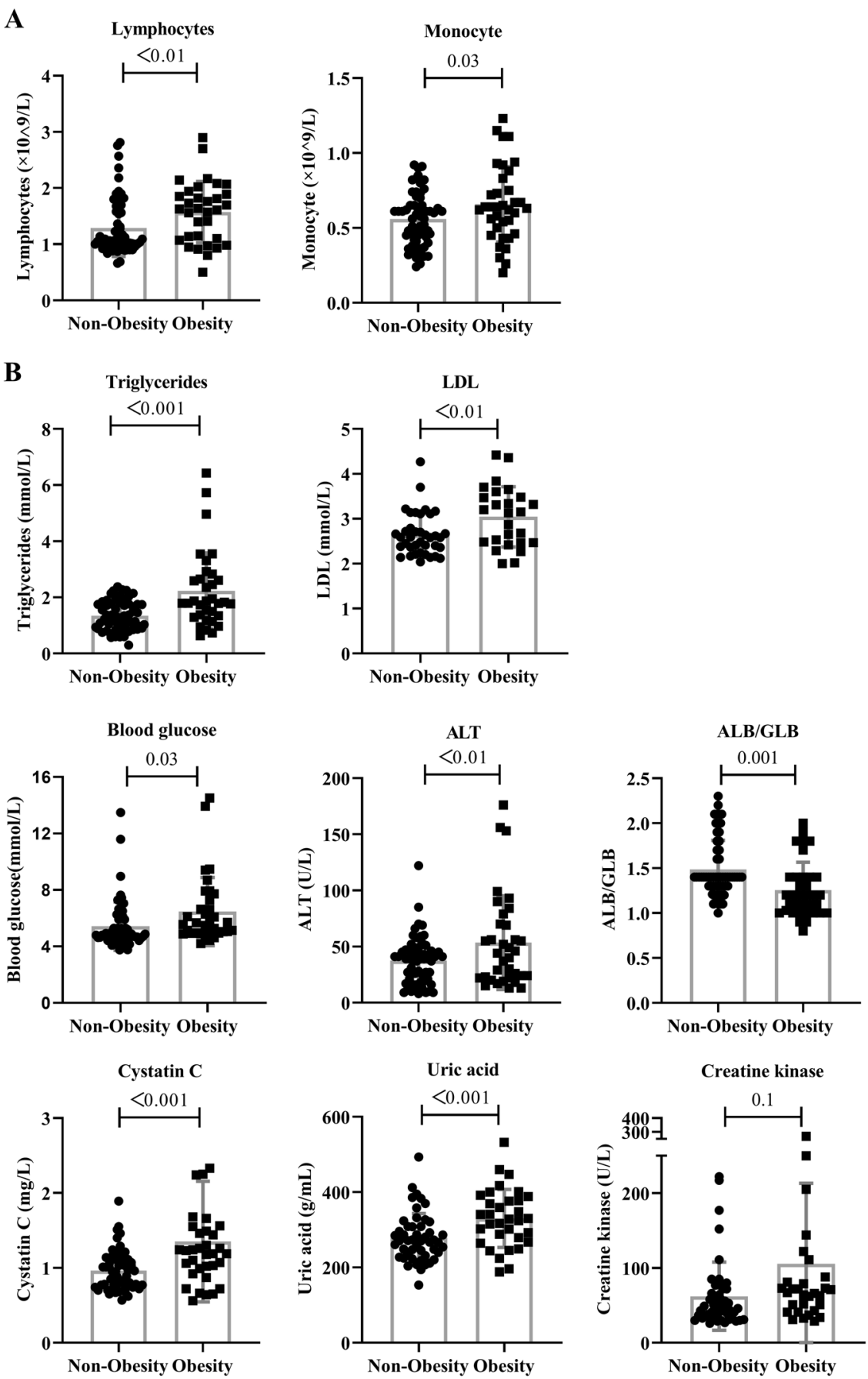

which are recruited to infected tissues and become tissue macrophages. These monocyte-derived macrophages perform phagocytosis in infected tissues [29]. Previous studies indicated the massive mononuclear cells infiltration in the lung, kidney, heart, lymph nodes of patients with COVID19 [30, 31], suggesting the important role of monocytes in the progression of disease. In the later stage of infection, SARS-CoV-2 can also initiate an adaptive T cell response, reflected by the changes in $\mathrm{T}$ cell differentiation and activation with markedly increased proportion of lymphocytes and upregulated activation markers on T cells. However, the levels of regulatory molecules such as PD-1, CTLA-4, and TIGIT, will were also increased during $\mathrm{T}$ cell responses, indicating the potential exhaustion of $\mathrm{T}$ cells in function [32]. During the innate and adaptive immune responses, inflammation-related molecules were also expressed. Our results illustrated that IL6, IL-4, ESR, and hsCRP proteins were much higher in COVID-19 patients with obesity compared to non-obesity patients, which is consistent with previous studies [7, 33]. Further more, higher levels of 
Fig. 4 Inflammation-related laboratory and coagulation function indicators examination results of the obesity patients and nonobesity patients. a Cytokine and detection and infectionrelated indicators examination results of the obesity and nonobesity patients. b Coagulation function indicators examination results of the obesity and nonobesity patients. $P<0.05$ was considered statistically significant.
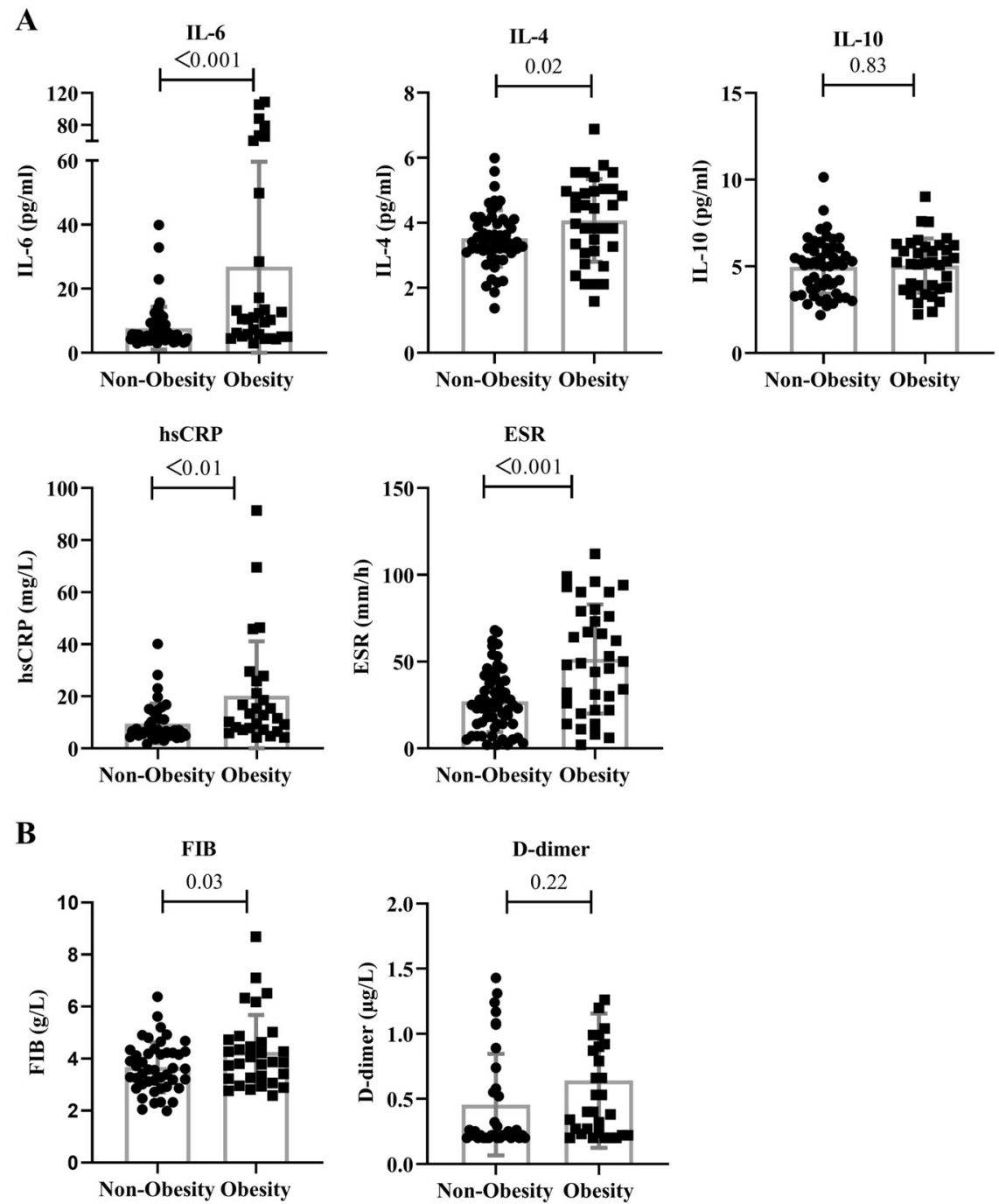

circulating inflammatory molecules could lead to severe damage in organs, such as lung, liver, and kidney, which is exactly consistent with significantly increased serum that the ALT, cystatin C, and severe lung pathological changes in obesity patients with COVID-19. The coagulation related factors were also detected in a higher level in obesity patients than those in non-obesity patients, suggesting a higher risk of thrombus formation including pulmonary embolism. This could explain the deterioration of the illness with sharp decrease of blood oxygen saturation in patients with COVID-19 in clinic. These results suggest that obesity patients with COVID-19 suffered more severe tissue damage and excessive immune responses. Thus obesity will affect the course of the disease and be associated with the prognosis of COVID-19 [10-12].

In summary, obesity contributes to clinical manifestations and may influence the progression and prognosis of COVID-19, with an increased risk of severe complications for subjects with obesity. Thereby, more attention needs to be paid to obesity patients in clinical treatment and later clinical follow-up, especially for those with additional other comorbidities. However, the exact mechanisms underlying obesity-related changes in the course of COVID-19 are not established yet, and needs to be resolved in the future.

Acknowledgements We would like to thank all members of Wuhan Union Hospital for helpful suggestions and discussions.

Funding This study was funded by the grants from the project of Thousand Youth Talents for DH; and from the China National Natural Science Foundation; and from China Postdoctoral Science Foundation (Nos. 31770983 and 81974249 to DH, No. 81601747 to SL, No. 2020T130040ZX to ZK).

\section{Compliance with ethical standards}

Conflict of interest All authors: No reported conflicts. All authors have submitted the ICMJE form for disclosure of potential conflicts of interest. Conflicts that the editors consider relevant to the content of the manuscript have been disclosed. 
Publisher's note Springer Nature remains neutral with regard to jurisdictional claims in published maps and institutional affiliations.

\section{References}

1. Chen N, Zhou M, Dong X, Qu J, Gong F, Han Y, et al. Epidemiological and clinical characteristics of 99 cases of 2019 novel coronavirus pneumonia in Wuhan, China: a descriptive study. Lancet. 2020;395:507-13.

2. Zhu N, Zhang D, Wang W, Li X, Yang B, Song J, et al. A novel coronavirus from patients with pneumonia in China, 2019. N Engl J Med. 2020;382:727-33.

3. Huang PL. A comprehensive definition for metabolic syndrome. Dis Models Mech. 2009;2:231-7.

4. Zhou P, Yang X-L, Wang X-G, Hu B, Zhang L, Zhang W, et al. A pneumonia outbreak associated with a new coronavirus of probable bat origin. Nature. 2020;579:270-3.

5. Hoffmann M, Kleine-Weber H, Schroeder S, Krüger N, Herrler T, Erichsen S, et al. SARS-CoV-2 cell entry depends on ace 2 and tmprss 2 and is blocked by a clinically proven protease inhibitor. Cell. 2020;181:271-80. e8.

6. Letko M, Munster V. Functional assessment of cell entry and receptor usage for lineage B $\beta$-coronaviruses, including 2019nCoV. 2020.

7. Zhang JJ, Dong X, Cao YY, Yuan YD, Yang YB, Yan YQ, et al. Clinical characteristics of 140 patients infected by SARS-CoV-2 in Wuhan, China. Allergy. 2020;75:1730-41.

8. Wang C, Horby PW, Hayden FG, Gao GF. A novel coronavirus outbreak of global health concern. Lancet. 2020;395:470-3.

9. Honce R, Karlsson EA, Wohlgemuth N, Estrada LD, Meliopoulos VA, Yao J, et al. Obesity-related microenvironment promotes emergence of virulent influenza virus strains. mBio. 2020;11: e03341-19.

10. Huttunen R, Syrjanen J. Obesity and the risk and outcome of infection. Int J Obes. 2013;37:333-40.

11. Jones CY, Hogan JW, Snyder B, Klein RS, Rompalo A, Schuman $\mathrm{P}$, et al. Overweight and human immunodeficiency virus (HIV) progression in women: associations HIV disease progression and changes in body mass index in women in the HIV epidemiology research study cohort. Clin Infect Dis. 2003;37:S69-80.

12. Falagas ME, Athanasoulia AP, Peppas G, Karageorgopoulos DE. Effect of body mass index on the outcome of infections: a systematic review. Obes Rev. 2009;10:280-9.

13. Muscogiuri G, Pugliese G, Barrea L, Savastano S, Colao A. Obesity: the "Achilles heel" for COVID-19? Metabolism. 2020;108:154251.

14. Louie JK, Acosta M, Samuel MC, Schechter R, Vugia DJ, Harriman $\mathrm{K}$, et al. A novel risk factor for a novel virus: obesity and 2009 pandemic influenza A (H1N1). Clinical Infect Dis. 2011;52:301-12.

15. Simonnet A, Chetboun M, Poissy J, Raverdy V, Noulette J, Duhamel A, et al. High prevalence of obesity in severe acute respiratory syndrome coronavirus-2 (SARS-CoV-2) requiring invasive mechanical ventilation. Obesity. 2020;28:1195-9.
16. Hussain A, Vasas P, El-Hasani S. Obesity as a risk factor for greater severity of COVID-19 in patients with metabolic associated fatty liver disease. Metabolism. 2020;108:154256-154256.

17. Rabec C, de Lucas Ramos P, Veale D. Respiratory complications of obesity. Arch Bronconeumol. 2011;47:252-61.

18. Klonoff DC, Umpierrez GE. COVID-19 in patients with diabetes: risk factors that increase morbidity. Metabolism. 2020:154224.

19. Pan WH, Yeh WT. How to define obesity? Evidence-based multiple action points for public awareness, screening, and treatment: an extension of Asian-Pacific recommendations. Asia Pac J Clin Nutr. 2008;17:370-4.

20. Consultation WE. Appropriate body-mass index for Asian populations and its implications for policy and intervention strategies. Lancet. 2004;363:157-63.

21. Low S, Chin MC, Ma S, Heng D, Deurenberg-Yap M. Rationale for redefining obesity in Asians. Ann Acad Med Singap. 2009;38:66-9.

22. Wang D, Hu B, Hu C, Zhu F, Liu X, Zhang J, et al. Clinical characteristics of 138 hospitalized patients with 2019 novel coronavirus-infected pneumonia in Wuhan, China. JAMA. 2020;323:1061-9.

23. Freedman KB, Back S, Bernstein J. Sample size and statistical power of randomised, controlled trials in orthopaedics. J Bone Joint Surg. 2001;83:397-402. British volume.

24. Guilherme A, Virbasius JV, Puri V, Czech MP. Adipocyte dysfunctions linking obesity to insulin resistance and type 2 diabetes. Nat Rev Mol Cell Biol. 2008;9:367-77.

25. Bremer AA, Devaraj S, Afify A, Jialal I. Adipose tissue dysregulation in patients with metabolic syndrome. J Clin Endocrinol Metab. 2011;96:E1782-8.

26. Dhurandhar NV, Bailey D. Interaction of obesity and infections. Obes Rev. 2015;16:1017-29.

27. Vieira-Potter VJ. Inflammation and macrophage modulation in adipose tissues. Cell Microbiol. 2014;16:1484-92.

28. Mehta P, McAuley DF, Brown M, Sanchez E, Tattersall RS, Manson JJ. COVID-19: consider cytokine storm syndromes and immunosuppression. Lancet. 2020;395:1033-4.

29. Merad M, Martin JC. Pathological inflammation in patients with COVID-19: a key role for monocytes and macrophages. Nat Rev Immunol. 2020;20:355-62.

30. Chen Y, Feng Z, Diao B, Wang R, Wang G, Wang C, et al. The novel severe acute respiratory syndrome coronavirus 2 (SARSCoV-2) directly decimates human spleens and lymph nodes. medRxiv. 2020: 2020.03.27.20045427.

31. Diao B, Wang C, Wang R, Feng Z, Tan Y, Wang H, et al. Human kidney is a target for novel severe acute respiratory syndrome coronavirus 2 (SARS-CoV-2) infection. medRxiv. 2020: 2020.03.04.20031120.

32. Zheng HY, Zhang M, Yang CX, Zhang N, Wang XC, Yang XP, et al. Elevated exhaustion levels and reduced functional diversity of $\mathrm{T}$ cells in peripheral blood may predict severe progression in COVID-19 patients. Cell Mol Immunol. 2020;17:541-3.

33. Han W, Quan B, Guo Y, Zhang J, Lu Y, Feng G, et al. The course of clinical diagnosis and treatment of a case infected with coronavirus disease 2019. J Med Virol. 2020;92:461-3. 